Instituto Internacional de Investigación y Desarrollo Tecnológico Educativo INDTEC, C.A. DOI: https://doi.org/10.29394/Scientific.issn.2542-2987.2020.5.16.1.20-39

OAI-PMH: $\underline{\text { http://www.indteca.com/ojs/index.php/Revista Scientific/oai }}$

Artículo Original / Original Article

\title{
Gestión de Información y Gestión de Comunicación en empresas que utilizan normas de comercio seguro
}

\author{
Autores: César Raúl Méndez Carpio \\ Universidad Católica de Cuenca, UCACUE \\ cmendezc@ucacue.edu.ec \\ Cuenca, Ecuador \\ https://orcid.org/0000-0003-0582-0107 \\ Natalia Adriana Palacios Montero \\ Universidad Técnica Particular de Loja, UTPL \\ npalacios@napalogistic.com \\ Cuenca, Ecuador \\ https://orcid.org/0000-0001-6625-2559
}

Resumen

Las empresas que forman parte de la cadena de suministros en el comercio exterior en Ecuador, mantienen su desarrollo organizacional basado en procesos regulados por normas de seguridad en la carga entre las que se encuentran las Basic Alliance for Secure Commerce (BASC) que, utilizan de forma transversal al tratamiento de la información y la comunicación, pero no las declaran de manera explícita. El objetivo de esta investigación fue sistematizar presupuestos teóricos acerca de los componentes de la Gestión de Información (GI); y la Gestión de la Comunicación (GC), que permitan plantear un modelo para su integración en procesos regulados por normas BASC como un recurso coadyuvante. La investigación desarrollada tiene un enfoque cualitativo y el método aplicado es el fenomenográfico apoyado de entrevistas semiestructuradas a expertos a fin de analizar sus procedimientos para tratar la información y la comunicación interna y externa de las empresas. Se encontró que las organizaciones no gestionan adecuadamente la información y la comunicación, pues no aplican principios infocomunicacionales, sino que se enfocan en tratamientos aislados, normativas y en muchos casos únicamente guiados por medios tecnológicos. En consecuencia, se plantea un primer modelo genérico infocomunicacional que permite integrar los componentes de información y la comunicación en beneficio de la gestión organizacional. exterior.

Palabras clave: gestión; información; comunicación; normativa; comercio

Cómo citar este artículo:

Méndez, C., \& Palacios, N. (2020). Gestión de Información y Gestión de Comunicación en empresas que utilizan normas de comercio seguro. Revista Scientific, 5(16), 20-39, e-ISSN: 2542-2987. Recuperado de: https://doi.org/10.29394/Scientific.issn.2542-2987.2020.5.16.1.20-39

Fecha de Recepción: 09-12-2019
Fecha de Aceptación: 30-03-2020
Fecha de Publicación: 05-05-2020 
OAI-PMH: http://www.indteca.com/ojs/index.php/Revista_Scientific/oai

Artículo Original / Original Article

\title{
Information Management and Communication Management in companies that use secure trade rules
}

\begin{abstract}
The companies that are part of the supply chain in foreign trade in Ecuador, maintain their organizational development based on processes regulated by cargo safety standards, among which are the Basic Alliance for Secure Commerce (BASC) that they use They are transversal to the treatment of information and communication, but they are not explicitly stated. The objective of this research was to systematize theoretical assumptions about the components of Information Management (GI); and the Communication Management (GC), that allow to propose a model for its integration in processes regulated by BASC norms as a contributing resource. The research carried out has a qualitative focus and the applied method is the phenomenographic supported by semi-structured interviews with experts in order to analyze their procedures for dealing with information and internal and external communication of companies. It was found that organizations do not adequately manage information and communication, since they do not apply info-communicational principles, but instead focus on isolated treatments, regulations and in many cases only guided by technological means. Consequently, a first generic info-communication model is proposed that allows integrating the information and communication components for the benefit of organizational management.
\end{abstract}

Keywords: management; information; communication; normative; foreign trade.

How to cite this article:

Méndez, C., \& Palacios, N. (2020). Information Management and Communication Management in companies that use secure trade rules. Revista Scientific, 5(16), 20-39, e-ISSN: 2542-2987. Recovered from: $\underline{\text { https://doi.org/10.29394/Scientific.issn.2542-2987.2020.5.16.1.20-39 }}$

Date Received:

09-12-2019
Date Acceptance:

30-03-2020
Date Publication: 05-05-2020 
Artículo Original / Original Article

\section{Introducción}

El sector empresarial en los Estados Unidos apoyado por varias empresas a nivel mundial, desarrolló un conjunto de ideas y preceptos que se fueron consolidando en normas, las cuales debían regular los procedimientos del manejo de la carga para facilitar el comercio exterior impidiendo que los grupos delictivos lo utilicen como fuente de contagio en la consecución de acciones ilícitas especialmente el tráfico ilegal de estupefacientes; dichas normas fueron estructuradas y denominadas con las siglas BASC (Basic Alliance for Secure Commerce).

A nivel mundial existen varios Capítulos BASC situados en distintos países que continuamente están hablando en sinergia para mejorar los procesos de aseguramiento del transporte de la carga comercial internacional y garantizando su seguridad frente al comercio ilícito y tráfico de mercancías que son los que generan grandes pérdidas en la economía mundial, fomentando la corrupción y el soborno.

Los capítulos BASC, se encuentran presentes en 11 países, entre los que figura el Ecuador y se suman a ello otros más, como Estados Unidos, Guatemala, México, Costa Rica, Panamá, República Dominicana, Venezuela, El Salvador, Perú y Colombia, con empresas miembros certificadas que, a pesar de no tener capítulos propios, han conseguido esta distinción en países con los que mantienen vínculos comerciales.

Las empresas que están inmersas en la cadena de suministros del comercio exterior en el Ecuador, regulan su gestión basándose en leyes, reglamentos y en estas normas que, buscan disminuir el tráfico de drogas y sustancias estupefacientes en sus cargas evitando la consumación de actividades ilícitas.

Las empresas que se encuentran dentro de la cadena de suministros del comercio exterior son: Exportadores e importadores, transportista de carga pesada, agentes afianzados y agentes de carga, navieras y puertos quienes 
OAl-PMH: http://www.indteca.com/ojs/index.php/Revista_Scientific/oai

Artículo Original / Original Article

requieren que la carga, llegue a su destino de manera correcta, segura y libre de contaminación. Por lo tanto, su gestión en el tratamiento de la información suministrada y sus procesos de comunicación deben ser muy eficientes para conseguir los objetivos organizacionales ya que de ello depende su desarrollo y supervivencia en el medio.

Las normas BASC, dentro de su desarrollo capitular tratan a la información y a la comunicación de forma muy superflua acentuándose únicamente en la seguridad de los equipos tecnológicos que sirven de apoyo a la gestión de la información y de la comunicación, pero no describen sus componentes de manera explícita que se traten de forma integral como se requiere.

Las empresas que forma parte de la cadena de suministros del comercio exterior, cada vez más se adscriben a los capítulos BASC apuntando a que sus cargas lleguen a su destino de manera correcta, segura y libre de contaminación. Por lo tanto, su gestión en el tratamiento de la información suministrada y sus procesos de comunicación deben ser muy eficientes para conseguir los objetivos organizacionales ya que de ello depende su desarrollo y supervivencia en el nicho de mercado en el que se desarrollen.

Dentro de este tipo de empresas, se ha analizado la necesidad de implementar sistemas de gestión certificados, pero la novedad existe al no haberse integrado los componentes claves de la gestión organizacional como son el manejo adecuado de la información y sus procesos de comunicación.

En este sentido, Alves y Duarte (2015): afirman que cuando se habla de ciencias de información y ciencias de la administración, se identifica una interdisciplinariedad ya que estas dos áreas son reconocidas como ciencias sociales aplicadas por tanto trascienden a cuestiones teóricas y epistemológicas acerca del territorio circunstancial de un producto informacional que propicia un desenvolvimiento organizacional.

Es sumamente necesario el conocimiento de las personas que usan la 
OAI-PMH: http://www.indteca.com/ojs/index.php/Revista_Scientific/oai

Artículo Original / Original Article

información para que realicen un manejo eficiente de los datos poniendo de por medio sus capacidades en función de los propósitos señalados en la misión de la organización.

Para Ponjuán (2004): "la gestión de la información es un proceso mediante el cual se obtienen, despliegan o utilizan recursos básicos (económicos, físicos, humanos, materiales) para manejar información dentro y para la sociedad a la que sirve" (pág. 17); este criterio está enfocado directamente con el desarrollo organizacional y su gestión.

Un concepto de gestión de información con un enfoque integral lo propone Rodríguez (2017), quien declara que:

La Gestión de Información se concibe como un proceso estratégico de planificación, organización, dirección y control, de forma eficaz y eficiente, de las estrategias, recursos, procesos, sistemas, productos, servicios, y demás capacidades informacionales existentes en una organización o en la sociedad, con el objetivo de mejorar el desempeño, la toma de decisiones, la adaptación al cambio, y la creación de fortalezas y ventajas competitivas por parte de los individuos, las organizaciones y la administración pública (pág. 12).

Al momento existe mucha información en línea, pero, ¿qué de toda esa información es la importante?. La respuesta a esta interrogante hará la diferencia entre una organización que gestione la información de manera eficiente frente a la que no lo hace. Por ello los objetivos organizacionales tienen que estar en concordancia con la gestión de la información la misma que permitirá tratar todos los procesos, procedimientos y actividades de calidad que realice el personal con un enfoque hacia la solución mediata e inmediata de los problemas consiguiendo un mejor desarrollo organizacional dentro de su sector y nicho de mercado.

Complementa, Zurkowski (1984): cuando anota acerca de la "Industria de la Información", se enfoca en el tratamiento de la información considerando la gestión en cuanto al desarrollo de productos y servicios dentro de los cuales 
menciona ciertos componentes como:

a). Servicios de contenido; en donde se incluyen bases de datos e índices

b). Paquetes de contenido; referente a libros, revistas, periódicos, cintas, videos.

c). Servicios de facilidades; servicios externos compartidos, transferencias y transacciones.

d). Tecnologías de Información; equipamiento electrónico, gráfico e impreso.

e). Tecnologías de integración; equipos de comunicación electrónica

f). Tecnologías de comunicación; equipos receptores punto a punto.

g). Canales de comunicación; transmisión de mensajes e información, canales cableados o de satélite.

h). Canales de difusión; a múltiples puntos a través de redes de tv, radio, entre otros.

En la empresa se deberían incluirse el tratamiento de todos estos componentes y ser incluidos como actividades de control e indicadores de gestión de la información consiguiendo mejoras en todos los frentes de la organización.

En referencia a la comunicación empresarial, Pérez (2014), señala que: "una organización humana es una realidad antropológica, es decir, una agrupación de personas unidas para hacer algo conjuntamente" (pág. 38). El concepto mencionado supone ideas muy genéricas en referencia a un sector de las organizaciones como lo es la empresa que requiere ser tratada con procesos de comunicación más desagregados y específicos.

En cambio, Fernández-Hernández (2012), tratan a la comunicación como un quehacer primordial en la organización y aseguran que además de que el personal esté calificado:

Debe estar localizado en el centro mismo del flujo de mensajes 
OAl-PMH: http://www.indteca.com/ojs/index.php/Revista_Scientific/oai

Artículo Original / Original Article

de la organización. Esto significa que tiene conocimiento de todos los tipos de flujos relevantes, aunque la cantidad de control directo que puede ejercer sobre esos flujos dependa de la disposición de la dirección para delegar la autoridad necesaria. Operando en el centro del flujo de mensajes de la organización, o cerca de él, el administrador de la comunicación puede servir como un enlace entre el resto de las unidades de trabajo y, por tanto, influir directamente en las características de los mensajes que esas unidades envían o reciben, tales como cantidad, calidad, oportunidad y forma (pág. 90).

La información en este contexto se constituye como elemento necesario puesto que es parte de un sistema y debe estar integrado a la gestión de la comunicación siendo tratados desde un esquema planificado tal que sus objetivos estratégicos sean los precursores del desarrollo de la organización con el propósito de cumplir la misión y visión establecidas.

Por otro lado, es imperativo señalar que toda organización debe analizar, controlar y potenciar la gestión de la comunicación basado en un análisis situacional que estudie los componentes de la comunicación organizacional que se detallan:

a). Los mensajes, que son un conjunto de signos, símbolos o señales que son transmitidos por los miembros de una organización y que tienen el propósito de informar.

b). Los canales, que son los espacios con ductos por donde se distribuyen los mensajes para llegar a su fin.

c). Los flujos, son las líneas de dirección comunicacional por donde se distribuyen los mensajes.

d). Los públicos, considerados como los que actúan en el proceso de la comunicación y pueden estar dentro y fuera de la organización y,

e). Las redes de comunicación, identificados como los medios técnicos que permiten la comunicación y que comparten puntos de análisis entre los 
miembros de la organización.

En el campo empresarial, la comunicación comparte dos ámbitos como son el interno y el externo por lo que debe haber armonía entre ellos para que fluya el proceso comunicacional.

Si se considera en primer lugar el ámbito interno, la comunicación cumple un papel muy importante ya que debe estar enfocada al cumplimiento de la misión, visión y los objetivos estratégicos organizacionales. En cuanto al ámbito externo, la comunicación debe centrarse en las relaciones con sus partes interesadas externas como son: el gobierno, la competencia, sus proveedores, los medios, etc.

Las empresas en tanto deben considerar sus relaciones de marketing, publicidad e información para conseguir posicionamiento en la sociedad en donde se desarrollan basándose en principios de gestión comunicacional e informacional.

En referencia a la caracterización de los principios de la gestión de la comunicación empresarial, al respecto Trelles (2001), indica que la comunicación organizacional:

Centra su atención en el análisis, diagnóstico, organización y perfeccionamiento de las complejas variables que conforman los procesos comunicativos en las organizaciones, a fin de mejorar la interrelación entre sus miembros, y entre estos y el público externo; fortalecer la identidad y mejorar el desempeño de las entidades (pág. 7).

Por su parte, Saló (2005), enfatiza que: en las organizaciones es necesario que la comunicación tenga una función de "carácter estratégico", que contenga la formulación de un plan de comunicación que se base en el análisis de necesidades, a través de un proceso de integración entre los objetivos, acciones de dirección y auditoría que, permita definir una estrategia global con posibles ventajas y beneficios. 
La implementación de este plan de forma operativa corresponde a su fase de ejecución en la que se deberá realizar primero un análisis y selección de los públicos para luego asignar un equipo de gestión que controle los sistemas de comunicación internos y externos realizando acciones, técnicas y soportes por áreas administrativas implicadas con los debidos presupuestos previstos.

La calendarización de actividades y tareas tanto principales como secundarias, así como el seguimiento continuo a cada una de ellas permite conseguir la retroalimentación de los resultados esperados para una mejora de la rentabilidad optimizando los recursos y mitigando los riesgos que puedan presentarse durante la implementación, desarrollo y conclusión del plan.

La pregunta planteada al iniciar este proceso y que ha permitido conseguir los resultados expuestos en la investigación es: ¿Cómo articular la gestión de información y la gestión de la comunicación en empresas de la cadena de suministros del comercio exterior ecuatoriano que aplican normas para evitar tráfico de drogas y consumación de actividades ilícitas?

Además, para la presente investigación, se ha considerado plantear el siguiente objetivo específico: caracterizar los componentes de la gestión de la información y la gestión de la comunicación en dichas empresas, lo cual permitirá mejorar la articulación de la gestión de información y la gestión de la comunicación que, junto a la gestión tecnológica, se puedan aplicar en el beneficio organizacional como complemento de los procesos de certificación BASC.

\section{Metodología (Materiales y métodos)}

El método de análisis aplicado, es el fenomenográfico con un enfoque netamente cualitativo, apoyado en un análisis documental tanto de las normas de seguridad en el comercio (normas BASC), como de los procesos y procedimientos caracterizados con los que cuentan la certificación de una 
muestra de las empresas de la cadena de suministros de exportación en el Ecuador

En relación con el tema de estudio, se han considerado la población o universo, a la distribución de tipos de empresas que forman parte de la cadena de suministros de exportación del comercio exterior y entre las que se notan; Navieras y Puertos, Transportistas, Agentes de carga internacional, agentes aduaneros y exportadores.

En correspondencia con las empresas Navieras, se encuentran registradas ante la Cámara Marítima del Ecuador 11 empresas y así mismo, se pueden citar una lista de 9 navieras más, que recalan sus buques en Ecuador y que se mantienen en la línea constante del comercio exterior de las cuales 5 se encuentran certificadas con normas BASC:

De los 6 puertos registrados, todos se encuentran certificados con normas BASC, lo que permite la continuidad de los procesos de exportación, en cambio, de las empresas de transporte o transportistas de carretera, se encuentran registrados 90 en el Ecuador, con una certificación BASC y solamente 4, en el capítulo Azuay, consideradas como empresas formales.

En referencia a los agentes de carga, se notaron en el registro, 36 empresas en los capítulos BASC, Ecuador y, solamente 1 en la ciudad de Cuenca capítulo Azuay, quienes tienen estrecha relación con los agentes de aduana que fungen 22 registrados en el país, aunque ninguno en el capítulo Azuay de la ciudad de Cuenca y, por último, en referencia a exportadores en el Ecuador, están registradas 735 empresas con certificados BASC, de las cuales en la ciudad de Cuenca, 11 se encuentran al momento con certificados BASC.

Con lo anotado y en cumplimiento al tipo de muestreo intencional con criterio del investigador, el proceso de selección ha considerado adoptar una muestra representativa de los tres capítulos que engloban el Ecuador como son: Capítulo Azuay, Capítulo Guayaquil y Capítulo Pichincha, quienes lo 
conforman son las empresas que han promovido la inclusión de los asociados de negocios en los mismos y que al no existir en proporciones equitativas y en algunos casos no tener presencia en Cuenca, Azuay, se ha considerado a nivel de país con el fin de trabajar con dos (2) representantes del Directorio; es decir, dos (2) de cada tipo, que forman la cadena de exportación: Navieras y Puertos, Transportistas, Agentes de carga, agente de aduanas y exportadores.

Se pudieron aplicar entrevistas semi estructuradas a expertos tanto en la gestión de información como en la gestión de la comunicación para afirmar criterios que permitan relacionar las dos categorías. Así mismo se aplicaron encuestas estructuradas a dirigentes de las organizaciones de la muestra que manejan los procesos y procedimientos que tienen estrecha relación con la aplicación de las normas BASC.

\section{Resultados (análisis e interpretación de los resultados)}

De los resultados conseguidos en la aplicación de las entrevistas a los diferentes expertos, se pudo concretar la construcción teórico-práctica de la categoría de análisis con criterios que permiten examinar las características diferenciadoras y el desarrollo de sus componentes.

Ante esto, Codina (2014), manifiesta que: "a pesar de los avances alcanzados en los últimos años en las comunicaciones electrónicas, la vía más utilizada por los directivos -inclusive incrementada según investigaciones recientes- es la comunicación oral” (pág. 184); con lo que complementa Moreno (2017): que "[...] los aspectos más importantes de las relaciones entre las personas se encuentra la comunicación, definida como el proceso donde se establecen lazos que implican conductas honestas [...]" (pág. 378); y, en concordancia con ello se han realizado acercamientos con diferentes expertos pudiendo destacar la siguiente información recabada:

La Gestión de la comunicación es concebida por los expertos 
OAl-PMH: http://www.indteca.com/ojs/index.php/Revista_Scientific/oai

Artículo Original / Original Article

consultados como un elemento de gran relevancia en las relaciones humanas y sociales, así también la gestión de la información debe tener la intención de diferenciar el tipo de información que vamos a necesitar en cada situación y donde encontrarla tomando en cuenta aquella información que le añada valor a lo que se genera dentro de la organización, convirtiéndose en una organización inteligente como lo llama Rodríguez (2016): "[...] es aquella que utiliza de forma intensiva información y conocimiento individual y organizacional favoreciendo el aprendizaje, la creación de conocimiento y la toma de decisiones para alcanzar de forma óptima su proyección estratégica [...]" (pág. 167).

Por otra parte, la Gestión de la Comunicación, es aquel proceso de construcción cíclica, en donde se establecen fases o etapas tales como la investigación, la planeación e implementación, para construir la evaluación organizacional. La naturaleza de las organizaciones no interesa, sino que el proceso o ciclo, busque la interacción entre sus etapas, fases y además entre las relaciones que deben existir en la Gestión de la Comunicación con la Gestión empresarial, en aras de potenciar la eficacia organizacional.

Gestionar la información tiene procesos comunes con la comunicación en la propia gestión empresarial, es considerar como los datos generados se gestionan en armonía entre los diferentes flujos informacionales, el contenido que se genera y, el uso de indicadores homogéneos tanto internos y externos concibiendo un análisis de impacto como control del sistema.

El momento de integrar a la información y a la comunicación con su gestión en la empresa, coinciden que estos elementos son estratégicos para su desarrollo puesto que consiguen el posicionamiento en el sector frente a sus competidores desarrollando la tan requerida mejora continua, la innovación y la consecución de la calidad demostrada.

Los procesos de información controlados por la empresa, son considerados activos de la misma, por lo tanto, su personal debe estar 
consciente de que no le corresponde como patrimonio personal sino como un recurso activo de la organización. La comunicación por otro lado, en el caso de las empresas y organizaciones, es la base estructural, pues la transmisión de información es una actividad diaria de gran importancia y en el nivel empresarial y organizacional mucho más. Al relacionarlas y hablar de infocomunicación, esta debe ser considerada como un motor integrado de cambio para las sociedades desarrolladas y, especialmente apoyada por la tecnología y el uso de la internet como una herramienta del quehacer diario, con el acceso inmediato y que propende la difusión y manejo de la información.

Una empresa que controle su sistema de Gestión de Comunicación y, su sistema Gestión de Información relacionados con los procesos y procedimientos, es una empresa que se mantiene en movimiento y se ve apoyada por la calidad para su desarrollo.

Los modelos que se manejen integrados, no se deben divorciar de las fases y etapas tanto de la gestión de comunicación como de la gestión de información imbricados en el ciclo de la gestión empresarial y sus principios. Los principios básicos deben considerar; la coordinación, la integralidad, la normación, entre otros que pueden ser principios básicos para buscar la legitimización de esa gestión empresarial. Tener una mirada transversal y transdisciplinar considerando la Implicación y participación del personal sería un muy buen aporte al desarrollo organizacional.

En relación con los componentes tanto de la Gestión de Comunicación y de la gestión de información, así como su integración, los resultados presentado en la tabla 1, producto de las respuestas tabuladas en la encuesta aplicada a los directivos y representantes del sistema de gestión de las empresas que conforman la cadena de suministros del comercio exterior. 
OAl-PMH: http://www.indteca.com/ojs/index.php/Revista_Scientific/oai

Artículo Original / Original Article

Tabla 1. Componentes de la GI y la GC (Resultado del estudio).

\begin{tabular}{|c|c|c|c|c|c|c|c|c|c|}
\hline \multicolumn{10}{|c|}{ GESTIÓN DE COMUNICACIÓN } \\
\hline \multicolumn{2}{|c|}{\begin{tabular}{|c|} 
¿La empresa da a \\
conocer a sus asociados \\
de negocios, las \\
políticas de \\
comunicación? \\
\end{tabular}} & \multicolumn{2}{|c|}{$\begin{array}{c}\text { ¿La planificación estratégica de } \\
\text { su empresa considera objetivos } \\
\text { vinculados con la gestión de la } \\
\text { comunicación y/o la gestión de } \\
\text { información? } \\
\end{array}$} & \multicolumn{2}{|c|}{$\begin{array}{c}\text { ¿Considera que los canales o } \\
\text { medios de comunicación } \\
\text { EXTERNA son los adecuados } \\
\text { con sus asociados de } \\
\text { negocios? }\end{array}$} & \multicolumn{2}{|c|}{$\begin{array}{l}\text { ¿Cree que la empresa } \\
\text { mantiene un control de los } \\
\text { mensajes entregados a sus } \\
\text { asociados de negocios? }\end{array}$} & \multicolumn{2}{|c|}{\begin{tabular}{|c|} 
¿La empresa realiza un \\
control y evaluación de la \\
comunicación organizacional \\
con miras a la mejora \\
continua?
\end{tabular}} \\
\hline SI & $30 \%$ & SI & $30 \%$ & SI & $90 \%$ & SI & $90 \%$ & SI & $50 \%$ \\
\hline NO & $70 \%$ & NO & $70 \%$ & NO & $10 \%$ & NO & $10 \%$ & NO & $50 \%$ \\
\hline \multicolumn{10}{|c|}{ GESTIÓN DE INFORMACIÓN } \\
\hline \multicolumn{2}{|c|}{$\begin{array}{l}\text { ¿La empresa cuenta } \\
\text { con un sistema de } \\
\text { información que } \\
\text { controla las }\end{array}$} & \multicolumn{2}{|c|}{$\begin{array}{l}\text { ¿Los procesos de la empresa } \\
\text { consideran la identificación, } \\
\text { recuperación, procesamiento, } \\
\text { análisis, almacenamiento y }\end{array}$} & \multicolumn{2}{|c|}{$\begin{array}{l}\text { ¿La empresa cuenta con } \\
\text { personal con competencias } \\
\text { adecuadas para el manejo de } \\
\text { la información generada }\end{array}$} & \multicolumn{2}{|c|}{$\begin{array}{l}\text { ¿El programa de } \\
\text { capacitación considera el } \\
\text { conocimiento de } \\
\text { competencias }\end{array}$} & \multicolumn{2}{|c|}{$\begin{array}{c}\text { ¿La empresa realiza un } \\
\text { control y monitoreo de los } \\
\text { resultados de la Gestión de } \\
\text { Información? }\end{array}$} \\
\hline SI & $20 \%$ & $\mathrm{SI}$ & $20 \%$ & $\mathrm{SI}$ & $90 \%$ & SI & $20 \%$ & SI & $20 \%$ \\
\hline NO & $80 \%$ & NO & $80 \%$ & NO & $10 \%$ & NO & $80 \%$ & NO & $80 \%$ \\
\hline & & \multicolumn{6}{|c|}{ GESTIÓN INFOCOMUNICACIONAL } & & \\
\hline & & \multicolumn{2}{|c|}{$\begin{array}{c}\text { ¿La alta dirección considera que } \\
\text { es importante una visión } \\
\text { integral entre el uso de la } \\
\text { información y el estado de la } \\
\text { comunicación? }\end{array}$} & \multicolumn{2}{|c|}{$\begin{array}{l}\text { ¿La empresa conforma } \\
\text { grupos de trabajo } \\
\text { multidisciplinario (GIC) para } \\
\text { abordar problemas } \\
\text { infocomunicacionales y sus }\end{array}$} & \multicolumn{2}{|c|}{$\begin{array}{c}\text { ¿La empresa verifica los } \\
\text { impactos de la información } \\
\text { y la comunicación en la } \\
\text { proyección estratégica } \\
\text { organizacional en función }\end{array}$} & & \\
\hline & & $\mathrm{SI}$ & $20 \%$ & $\mathrm{SI}$ & $20 \%$ & SI & $10 \%$ & & \\
\hline & & NO & $80 \%$ & NO & $80 \%$ & NO & $90 \%$ & & \\
\hline
\end{tabular}

Fuente: Los Autores (2019).

El $70 \%$ de las empresas encuestadas, afirman dar a conocer sus políticas de comunicación a los miembros de la organización, aunque apenas el $30 \%$ de ellas, vinculan su planificación estratégica con la gestión de la comunicación y la gestión de información. El 90\% de empresas consultadas afirman que los canales o medios de comunicación externa son los adecuados con sus asociados de negocios, solamente una de ellas dice no controlarlo.

Así mismo coinciden el $90 \%$ de empresas en afirmar que mantienen control de los mensajes entregados a sus asociados de negocios y, se muestra criterios divididos al $50 \%$ de las empresas que dicen controlar y evaluar la comunicación organizacional con miras a la mejora continua.

En referencia a la unidad de análisis gestión de información, del total de empresas encuestadas, el $20 \%$ dicen controlar las necesidades y fuentes de información relevante y están de acuerdo al 100\% luego de la explicación que lo deberían realizar. El $80 \%$ de ellas no tratan a la información como un sistema ya que contestan no seguir el ciclo de identificación, recuperación, 
procesamiento, análisis, almacenamiento y diseminación del uso adecuado de la información, pero están de acuerdo en que deben cumplir el sistema.

Se puede observar además que, la mitad de empresas estudiadas, dicen que disponen del personal con competencias adecuadas para el uso de la información y están de acuerdo así mismo luego de una inducción que, deberían incorporarlo a su gestión. Es notorio que apenas el $20 \%$ de las empresas tienen un programa de capacitación que considere el conocimiento de competencias informacionales y ese mismo porcentaje bajo, afirman controlar y monitorear los resultados de la Gestión de información, demostrando un acuerdo en que estas actividades deberían ser controladas en su planificación.

En referencia a la integración de las categorías de análisis; gestión de información y gestión de la comunicación, se observa que no las gestionan de manera integrada, pues solamente un $20 \%$ de las empresas de la cadena de suministros del comercio exterior, conforman grupos con el Gestor de Información y Comunicación (GIC), aunque están de acuerdo en que es necesario considerar la conformación de estos grupos multidisciplinarios como un todo administrativo.

Al final se observa que es mínima la verificación de impactos $10 \%$ de la información y la comunicación en la proyección estratégica organizacional en función de la mejora infocomunicacional.

\section{Conclusiones}

En la sociedad actual, llamada también la sociedad del conocimiento y la información, es imperante el nivel de educación del personal de la empresa que posea las suficientes destrezas y habilidades en los campos de la información y la comunicación con el fin de emprender cualquier cambio. Por ello, como señalan Castro, Pérez, Pérez y Caldera de Sánchez (2019): se "[...] ha demostrado que la educación se ha constituido en una herramienta para la 
OAI-PMH: http://www.indteca.com/ojs/index.php/Revista_Scientific/oai

Artículo Original / Original Article

transformación social y el mejoramiento sostenido de la calidad de vida y, en la misma esencia del hombre en su condición de ciudadano" (pág. 106); apoyando los procesos de alfabetización tan necesarios en la gestión organizacional. Basados en esta afirmación se han podido sistematizar los presupuestos teóricos que definen tanto a la gestión de información como a la gestión de la comunicación con el fin de hibridarlos en un modelo preliminar.

Figura 1. Modelo de Gestión Infocomunicacional para el Desarrollo Empresarial.

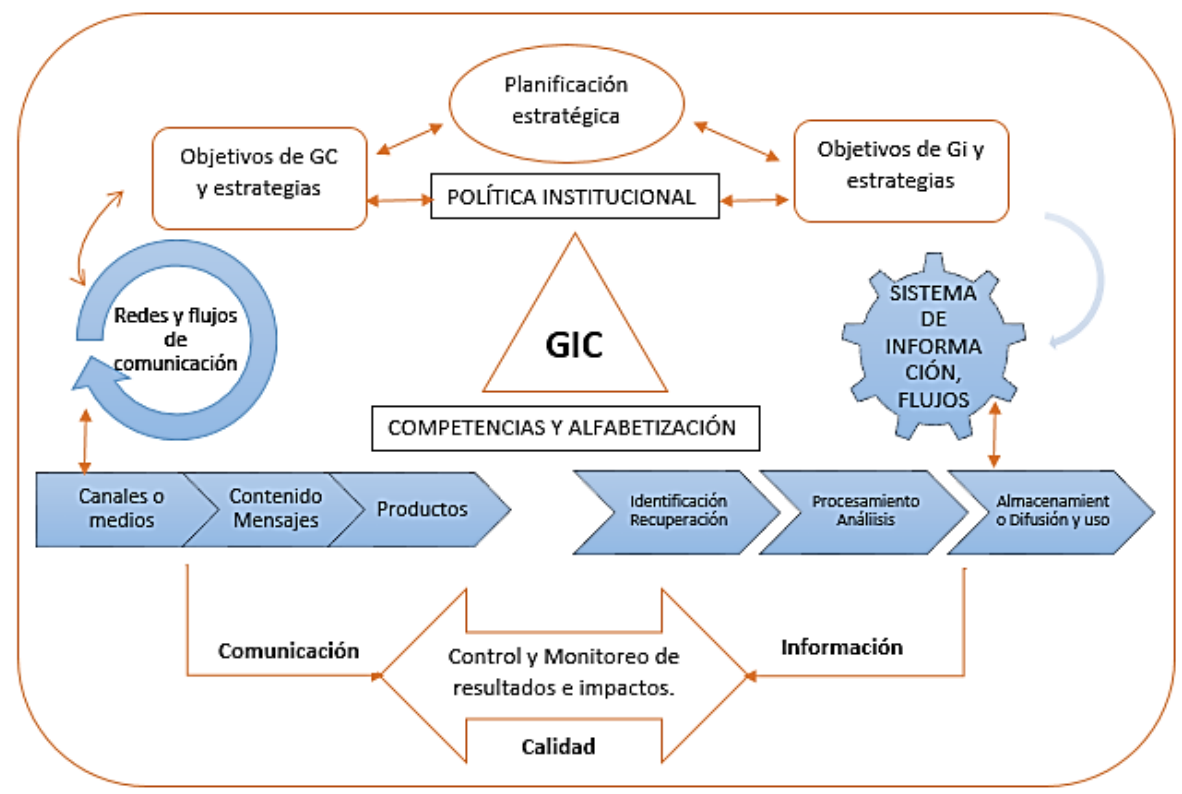

Fuente: Los Autores (2019).

Luego de ello y, una vez analizados los datos obtenidos en la ejecución de la metodología aplicada producto de entrevistas a expertos, observación in situ y de encuestas aplicadas a los miembros responsables de la gestión por procesos, directivos y/o auditores BASC y, en concordancia con el marco teórico conceptual, se confirma la desintegración de las categorías de análisis en la mayoría de empresas que conforman la cadena de suministros del comercio exterior en función incluso de los aportes de los autores analizados 
durante el desarrollo del presente trabajo de investigación; por lo cual en conclusión se propone un modelo infocomunicacional que integra tanto la gestión de información como la gestión de la comunicación en beneficio del desarrollo empresarial de dichas organizaciones que utilizan las normas BASC, las cuales no explicitan entre sus capítulos normativos, los componentes que se detallan en la figura 1.

Los componentes que se mencionan y se han podido graficar, permitirán integrarse para que una vez validados por expertos, sean expuestos de forma explícita en las nuevas versiones de las normas BASC como mejora en el desarrollo organizacional.

\section{Referencias}

Alves, C., \& Duarte, E. (2015). A relação entre a Ciência da Informação e a Ciência da Administração. Transinformação, 27(1), 37-46, e-ISSN 0103-3786. Recuperado de:

http://dx.doi.org/10.1590/0103-37862015000100004

Castro, J., Pérez, J., Pérez, J., \& Caldera de Sánchez, B. (2019). Las TIC's como Herramienta de Apoyo de la Estrategia Instruccional del Docente Universitario. Revista Scientific, 4(12), 104-126, e-ISSN: 2542-2987. Recuperado de:

https://doi.org/10.29394/Scientific.issn.2542-2987.2019.4.12.5.104-126

Codina, A. (2014). Habilidades Directivas. La Habana, Cuba: Editorial Academia.

Fernández-Hernández, Z. (2012). El comunicador en la gestión integral de la comunicación. Ciencia en su PC, (2), 87-99, e-ISSN: 1027-2887. Recuperado de: http://www.redalyc.org/articulo.oa?id=181324082007 Moreno, R. (2017). Líneas Estratégicas de Comunicación en el Desarrollo de Habilidades Gerenciales y Humanas. Revista Scientific, 2(Ed. Esp.), 376-393, e-ISSN: 2542-2987. Recuperado de: 
OAI-PMH: http://www.indteca.com/ojs/index.php/Revista_Scientific/oai

Artículo Original / Original Article

https://doi.org/10.29394/scientific.issn.2542-2987.2017.0.0.20.376-393

Pérez, J. (2014). Fundamentos de la Dirección de Empresas. Madrid, España: Ediciones Rialp, S.A.

Ponjuán, G. (2004). Gestión de información: dimensiones e implementación para el éxito organizacional. ISBN: 987-96536-6-1. Rosario, Argentina: Nuevo Parhadigma.

Rodríguez, Y. (2017). Rutas para una gestión estratégica y articulada de la información y la comunicación en contextos organizacionales. alcance: Revista Cubana de Información y Comunicación, 6(14), 3-31, e-ISSN: 2411-9970. Recuperado de:

http://www.alcance.uh.cu/index.php/RCIC/article/view/105

Rodríguez Y. (2016). Concepción estratégica de la Gestión de Información y del Conocimiento para organizaciones inteligentes. Bibliotecas: Anales de Investigación, 12(2), 165-181, ISSN: 0006-176X; e-ISSN: 1683-8947. Recuperado de:

https://dialnet.unirioja.es/servlet/articulo?codigo $=5766703$

Saló, N. (2005). Aprender a comunicarse en las organizaciones. ISBN: 84493-1771-1. Barcelona, España: Ediciones Paidós Ibérica, S.A.

Trelles, I. (2001). Comunicación organizacional. Cuba: Editorial Universitaria Félix Varela.

Zurkowski, P. (1984). Integrating America's infostructure. Jasist: Journal of the American Society for Information Science and Technology, 35, 170178, ISSN: 2330-1635; e-ISSN: 2330-1643. Recovered from: https://doi.org/10.1002/asi.4630350310 


\section{Artículo Original / Original Article}

\section{César Raúl Méndez Carpio \\ e-mail: cmendezc@ucacue.edu.ec}

Nacido en Cuenca, Ecuador, el 22 de febrero del año 1971.

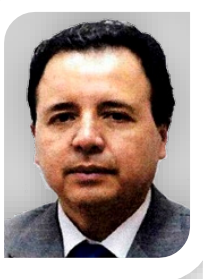

Dr. En Ciencias de la Educación, especialidad Ordenadores en la Universidad Católica de Cuenca (UCACUE); Magister en Docencia y Currículo para la Educación Superior en la Universidad Técnica de Ambato (UNITA); Magister en Administración de Empresas en la Universidad del Azuay (UDA); Actualmente me desempeño como Jefe de Investigación en el Colegio Militar "Abdón Calderón" y como docente en la Universidad Católica de Cuenca (UCACUE), en las Asignaturas: Estadística Aplicada, Tecnologías de Información y Comunicación. 
OAI-PMH: http://www.indteca.com/ojs/index.php/Revista_Scientific/oai

\section{Artículo Original / Original Article}

\section{Natalia Adriana Palacios Montero \\ e-mail: npalacios@napalogistic.com}

Nacida en Cuenca, Ecuador, el 21 de septiembre del año

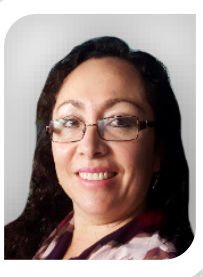
1977. Me gradué en el Colegio Manuela Garaicoa de Calderón, en la especialidad de Secretariado Español; soy Auditora de normas BASC para el capítulo Azuay; Actualmente me desempeño como General Manager en la Empresa NAPALOGISTIC IFF, que es un Freight Forwarders o Agente de Carga Internacional. 